\title{
Discordance of Histo-pathological Diagnosis of Patients with Soft Tissue Sarcoma Referred to Tertiary Care Center
}

\author{
Sameer Rastogi ${ }^{1}$, Aditi Aggarwal ${ }^{2}$, Sorun Shishak ${ }^{3}$, Adarsh Barwad ${ }^{4}$, Ekta \\ Dhamija $^{5}$, Rambha Pandey ${ }^{3}$,Asit Ranjan Mridha ${ }^{4}$, Venkatesan Sampath kumar ${ }^{6}$, \\ Shah Alam Khan ${ }^{6}$, Suryanarayana S.V. Deo ${ }^{7}$, Mehar Chand Sharma ${ }^{4}$
}

${ }^{1}$ Department of Medical Oncology, All India Institute of Medical Sciences (AIIMS), New Delhi, India. ${ }^{2}$ Department of Radiation Oncology, National Cancer Institute-India, AIIMS, Jhajjar, India. ${ }^{3}$ Department of Radiation Oncology, All India Institute of Medical Sciences (AIIMS), New Delhi, India. ${ }^{4}$ Department of Pathology, All India Institute of Medical Sciences (AIIMS), New Delhi, India. ${ }^{5}$ Department of Radiodiagnosis, All India Institute of Medical Sciences (AIIMS), New Delhi, India. ${ }^{6}$ Department of Orthopaedics, All India Institute of Medical Sciences (AIIMS), New Delhi, India. ${ }^{7}$ Department of Surgical Oncology, All India Institute of Medical Sciences (AIIMS), New Delhi, India.

\begin{abstract}
Background: Reaching the correct histo-pathological diagnosis of soft tissue sarcomas (STS) is a great challenge and is cornerstone for treatment planning. Need of expertise for diagnosis is limited due to the lack of dedicated expert sarcoma pathologists and oncologists in India. In this study, we highlight the pattern of pathological diagnosis and its accuracy outside specialist centre. Methods: We performed retrospective analysis of all patients referred to us with a clinical or histopathologic diagnosis of STS over the period January 2016 to December 2017. According to the protocol, all patients had a review of histopathology diagnosis from our institute. The tissue blocks if available were reviewed and a fresh biopsy was performed when required. The histopathologic diagnosis was also reviewed in the joint clinic, giving clinics-radiological inputs to sarcoma pathologists. For the patients with outside diagnosis and discordant report, we divided them into major discrepancy (including change of diagnosis of sarcoma to benign or other histological entity that could potentially change the treatment plan) or minor discrepancy (like mild change in grade or histopathological diagnosis not affecting the treatment plan). Statistical analysis was done by SPSS ver 23. Results: There were 149 patients with median age of 36 years (range $14-77$ years), and 93 patients (62.4\%) were males. About 57\% ( 85 cases) of patients had localized disease. Most common subtypes were synovial sarcoma (16\%), liposarcoma (9\%), soft tissue ewings sarcoma (9\%), MPNST $(9 \%)$, leiomyosarcoma (8\%), and undifferentiated pleomorphic sarcoma (8\%). Of 149 patients, $47(31.5 \%)$ had not been worked up outside by immunohistochemistry or other molecular studies and thus comparison was not possible; while 4 patients couldn't retrieve blocks and repeat biopsy could not be performed. Of 97 patients (biopsy $=84$, FNAC $=13$ ) who had diagnosis from outside, $37 \%$ had major discrepancy and $24 \%$ had minor discrepancy as compared with our diagnosis from the sarcoma specialists. Univariate analysis revealed that the major discrepancy was more in non-extremity than extremity STS $(p=0.003)$. Conclusion: Histopathologic diagnosis in more than half of patients referred from outside centers was discordant with respect to the diagnosis of our centre with major implications on $37 \%$ of cases. We believe this is due to the lack of sarcoma pathology experts, and they are virtually non-existent in the multidisciplinary clinic set up outside the tertiary care centres.
\end{abstract}

Keywords: Soft tissue sarcoma- histopathology- pathology- sarcoma- discordance

Asian Pac J Cancer Care, 4 (4), 119-123

\section{Introduction}

Soft tissue sarcoma (STS) is an exceedingly complex disease mainly because of its heterogeneity, rarity, and
Submission Date: 04/12/2019 Acceptance Date: 07/09/2019

ability to arise from different locations in the body. The treatment of soft tissue sarcoma essentially requires dedicated sarcoma pathologists, surgeons, medical oncologist, radiation oncologist, palliative care team etc.

Corresponding Author:

Dr. Sameer Rastogi

Department of Medical Oncology, All India Institute of Medical Sciences (AIIMS), New Delhi.

Email: samdoc_mamc@yahoo.com 
as a part of multi-disciplinary team [1]. Expert pathology unarguably is the cornerstone of the management of STS. Pathology of sarcoma is far more challenging than epithelial tumors because of rarity and multitude of subtypes [2]. The advent of specialized molecular testing has further complicated the scenario. The rarity of disease and non-specialized pathology reporting might lead to inappropriate treatment and thus poor outcomes [3-5].

Most of the data, literature and guidelines regarding pathology of sarcoma come from the western studies. In a report from Royal Marsden Centre, Thway et al [6] compared sarcoma unit's histopathology reports with referring reports on 349 specimens in patients with suspected or proven soft tissue sarcoma. Major discrepancy was defined by the discordance that could lead to change in treatment; while, minor discrepancy was defined as discordance that was not thought to provoke significant treatment change. On comparison from outside report, diagnostic concordance was found in $73.4 \%(\mathrm{n}=349)$ of cases with minor diagnostic discordance in $15.7 \%$ of cases, and major discordance in $10.9 \%$ of cases. Furthermore, the cause of this discrepancy was the different opinion of general or non-soft tissue sarcoma pathologists and pathologists at the specialist unit [6]. The rate of error in the diagnosis of soft tissue sarcoma in literature varies widely between $25-40 \%$ which further gives impetus to the fact that the review should be done by a bone and soft tissue pathologist, who evaluates soft tissue sarcoma regularly [6-9].

We believe that this discrepancy could be more in developing countries like India due to various factors. Thus a strategy should be developed to review all the cases or suspected cases of sarcoma from dedicated sarcoma pathologists. We conducted retrospective analysis of patients referred to our sarcoma clinic with an outside diagnosis and compared with our diagnosis that was made by specialists in bone and soft tissue pathology.

\section{Materials and Methods}

We retrospectively analysed patients referred to us with a diagnosis of soft tissue sarcoma (STS) in dedicated Sarcoma Medical Oncology clinic in All India Institute of Medical Sciences from January 2016 to December 2017. Many times, patients are referred to us from other clinics like hematolymphoid or pulmonary departments when the pathology unsuspectingly turns out to be sarcoma (intrainstitutional referral) or we get patients referred from outside directly when the outside biopsy is suggestive of sarcoma. As per institutional protocol we review histopathology diagnosis of outside institute by our bone and soft tissue pathology experts in all cases irrespective of that being reported from outside sarcoma specialized centers. If blocks were available, they were reviewed, and when necessary, a repeat biopsy was taken. Repeat biopsy was done in all patients who had only FNAC diagnosis from outside. The data for the study were collected during the course of common clinical practice. Signed informed consent was obtained from each patient for any procedure.

For the cases diagnosed outside and had discordant reports as compared to our (institutional) histopathology diagnosis we divided them into major discrepancy (including change of subtype/grade of sarcoma effecting further treatment, change to histopathology other than sarcoma or change to benign histopathology) or minor discrepancy like change in grade/subtype not affecting further management plan. When actual diagnosis was one of the differential diagnoses of the outside provisional report we considered that as minor discrepancy. For example, if the outside report for soft tissue ewings sarcoma was given as? round cell tumor? ewings sarcoma then we included those cases in minor discrepancy category.

\section{Sarcoma Pathology reporting in AIIMS}

We have two committed sarcoma pathologists at our institute and there is weekly pathology meeting during which all atypical/ rare cases are discussed and correlated with radiology as well. Molecular testing like translocation study was done wherever available and, in cases when the test was performed in outside laboratory the cost was borne by patient.

\section{Statistical Analysis}

All statistical analyses were performed using the Statistical Package for the Social Sciences Inc., version 18.0 for Windows. Nominal data are provided as number (\%) and continuous data as median (range). Univariate analysis was done to delineate the characteristics of sarcoma in which discrepancy was there.

\section{Results}

There was total of 149 soft tissue sarcoma patients registered during this time. Patient characteristics are shown in Table 1 (median age, male:female, range, metastatic, truncal, extremity, non-extremity). Of 149 patients, 102 patients had diagnosis from outside; while 47 patients were not worked up outside for providing diagnosis. Out of 102 cases, 4 patients refused to undergo a repeat biopsy. Hence, treatment was continued on the basis of previous diagnosis; while data were unavailable in another 1 case.

Diagnostic correlation was done in 97 patients who had a diagnosis available from both outside and our centre. Outside diagnosis was given on biopsy in $86.5 \%$ (84 cases) and FNAC in $13.4 \%$ (13 cases) of cases. Immunohistochemistry report in outside diagnosis was available in $27.8 \%$ (27 cases) of patients. Complete data were available in 81 patients, $76.5 \%$ (62 cases) of them were worked up in private centre, while 19 patients in government set up (medical college). Grade of sarcoma in outside report was given only in $13 \%$ of patients. Major discrepancy was present in $37.1 \%$ (36 patients); while, minor discrepancy was present in $23.7 \%$ (23 patients) of cases. Of those patients who had immunohistochemistry report from outside the major discrepancy was seen in $33 \%$ ( 9 cases) of patients.

We further divided major discrepancy into 
Table 1. Showing Characteristics of All Patients

\begin{tabular}{lc}
\hline Characterstics & $(\mathrm{n}=149)$ \\
\hline Median Age & 36 years \\
Age Range & $14-77$ years \\
Sex & \\
$\quad$ Males & 93 \\
Females & 56 \\
Extent of disease & \\
Metastatic & 64 \\
Non metastatic & 85 \\
Subtypes (after review from our institute) & \\
Synovial sarcoma & $25(16.8 \%)$ \\
Liposarcoma & $13(8.7 \%)$ \\
Malignant Peripheral Nerve Sheath Tumor & $13(8.7 \%)$ \\
Soft tissue Ewings Sarcoma & $13(8.7 \%)$ \\
Leiomyosarcoma & $12(8.1 \%)$ \\
Pleomorphic undifferentiated sarcoma & $12(8.1 \%)$ \\
Gastrointestinal stromal tumor & $9(6 \%)$ \\
Others & $52(34.8 \%)$ \\
Diagnostic test done outside (biopsy or FNAC) & \\
Yes & 102 \\
No & \\
Extremity vs non Extremity primary & $8(5.4 \%)$ \\
Extemity & \\
Non extremity & \\
Retroperitoneum & \\
Trunk & $23(14 \%)$ \\
Visceral & \\
Head and neck & \\
\hline
\end{tabular}

a. Sarcoma outside to some other tumor inside $(n=3$, $8.3 \%)$

b. Benign or other histopathology outside to sarcoma in our institute $(\mathrm{n}=15,41.6 \%)$

c. Change in histopathology diagnosis from one sarcoma to another sarcoma type $(n=18,50 \%)$

a. Sarcoma outside to another tumor inside

1. Undifferentiated sarcoma to lymphoma (thigh mass)

2. Undifferentiated sarcoma to lymphoma (chest wall mass with paraspinal component)

3. Liver ewings sarcoma to Neuroendocrine tumor

b. Benign and other histopathology to sarcoma in our institute

1. Arm fibromatosis to synovial sarcoma

2. Parapharyngeal adenocarcinoma to MPNST

3. Lymphoma of pelvis to ewings sarcoma (translocation proven)

4. Granulosa cell tumor ovary to ewings sarcoma ovary (translocation proven)

5. Tuberculosis knee to clear cell sarcoma
Table 2. Univariate Analysis for the Major Discrepancy in Pathology in Soft Tissue Sarcoma

\begin{tabular}{lcc}
\hline Characteristics & $\begin{array}{c}\text { Percentage of } \\
\text { major discrepancy }\end{array}$ & P value \\
\hline $\begin{array}{l}\text { Types of outside hospital } \\
\text { Private }\end{array}$ & $35.7 \%$ & 0.867 \\
$\begin{array}{l}\text { Public } \\
\text { Location of the tumor }\end{array}$ & $40 \%$ & \\
Extremity & & \\
Non extremity & $19.4 \%$ & \\
Type of sample done outside & $48 \%$ & 0.005 \\
Biopsy & & \\
FNAC & $34.5 \%$ & \\
Age at presentation & $53.8 \%$ & 0.18 \\
Less than or =40 years & & \\
More than 40 years & $41 \%$ & \\
\hline
\end{tabular}

6. Hemangioma to ewings sarcoma thigh

7. Kidney NHL changed to kidney ewings sarcoma

8. Lymphoma to mediastinal liposarcoma

9. Spindle cell thymoma to synovial sarcoma

(X 18 positive)

10. Schwannoma to MPNST

11. Adenocarcinoma lung to pulmonary intimal sarcoma

12. Benign spindle cell tumor of lung to pulmonary myxoid sarcoma

13. Germ cell tumor to rhabdomyosarcoma

14. Plexiform neurofibroma to synovial sarcoma

15. Small cell carcinoma to synovial sarcoma

c. One sarcoma subtype to another sarcoma subtype

1. Abdominal myxofibrosarcoma to abdominal rhabdomyosarcoma

2. Pleomorphic sarcoma to MPNST

3. Pleomorphic rhabdomyosarcoma to pleomorphic leiomyosarcoma

4. Spindle cell carcinoma to rhabdomyosarcoma

5. Scalp rhabdomyosarcoma to Alveolar soft part sarcoma

6. Angiosarcoma to dendritic follicular cell neoplasm

7. Synovial sarcoma to epitheloid sarcoma

8. Extraskeletal chodrosarcoma to extraskeletal osteosarcoma

9. Fibrosarcoma to GIST

10. Pleomorphic undiffentiated sarcoma to GIST

11. Myofibroblastic tumor to GIST

12. MPNST to GIST

13. Rhabdomyosarcoma to leiomyosarcoma

14. Pleomorphic undifferentiated sarcoma to solitary fibrous tumor

15. Dediffentiated liposarcoma to pleomorphic liposarcoma

16. Fibrosarcoma to solitary fibrous tumor

17. Fibrosarcoma to synovial sarcoma

18. Pleomorphic liposarcoma to Well differentiated liposarcoma

Minor discrepancy was all due to change in histopathology from one sarcoma to another sarcoma subtype except in one case which was due to change 
Table 3. Showing Minor Discrepancy Detail

\begin{tabular}{ll}
\hline 1. Ewings sarcoma/ synovial sarcoma ? & 1. Ewings sarcoma soft tissue \\
2. Small round cell tumor? & 2. Desmplastic round cell tumor \\
3. Round cell tumor? Sarcoma & 3. Low grade sarcoma \\
4. Pleomorphic sarcoma NOS & 4. Myxoid liposarcoma \\
5. Undifferentiated sarcoma & 5. Leiomyosarcoma \\
6. Undifferntiated sarcoma & 6. Leiomyosarcoma \\
7. Round cell tumor (? Neuroblastoma/ ? Ewings sarcoma) & 7. Ewings sarcoma soft tissue \\
8. Synvial sarcoma/ ewings sarcoma malignant mesenchymal tumor & 8. Soft tissue ewings sarcoma \\
9. ? Sarcoma & 9. Undifferentiated sarcoma \\
10. Ewings/ synovial sarcoma & 10. Ewings sarcoma soft tissue \\
11. Mesenchymal tumor (? Benign/ ? malignant) & 11. Fibromatosis \\
12. MPNST / SFT (solitary fibrous tumor) & 12. MPNST \\
13. ? Round cell tumor & 13. Soft tissue ewings sarcoma \\
14. Spindle cell tumor & 14. Synovial sarcoma \\
15. ? round cell tumor & 15. Ewings sarcoma \\
16. Spindle cell sarcoma & 16. Synovial sarcoma \\
17. Spindle cell sarcoma & 17. Myxofibrosarcoma \\
18. Spindle cell sarcoma & 18. Pleomorphic undifferentiated sarcoma \\
19. Myxofibrosarcoma & 19. Dedifferentiated liposarcoma \\
20. Myxoid liposarcoma grade 1 & 20. Myxoid liposarcoma grade 2 \\
21. Non malignant mesenchymal tumor ? DFSP & 21. Dermatofibrosarcoma protuberans \\
22. Soft tissue sarcoma & 22. MPNST \\
23. Leiomyosarcoma & 23. Undifferentiated sarcoma \\
\hline
\end{tabular}

in the grade (this may be due to very few numbers of cases where grade was given in outside report) and are summarized in Table 3.

In univariate analysis the major discrepancy was correlated with the factors such as location of tumors, type of sample (FNAC vs biopsy), age at presentation 40 years or less vs more than 40 years, reported outside in private vs government centre. The only factor which showed statistical significance was location of tumor (Table 2), and the discrepancy was significantly more in non-extremity tumors. Though the percentage was relatively higher in patients diagnosed by FNAC, yet it was not statistically significant. Besides, the type of outside institute where patient had been worked up was immaterial.

\section{Discussion}

This paper underscores several important aspects in the diagnosis of soft tissue sarcoma. Firstly, the younger presentation in our setting which could be due to relatively younger population pyramid in India. Secondly, most of the patients $102 / 149(68 \%)$ before presenting to us already had diagnostic workup elsewhere including biopsy or FNAC. There were 13 patients $(12.7 \%$ of all with any tissue workup outside) in whom FNAC was done outside. FNAC is not recommended in soft tissue sarcoma and might be responsible for delay in the diagnosis and diagnostic discrepancy in many cases leading to inadequate information and thus treatment [10].

The main purpose of this study is to discuss the importance of highly specialized skills in pathology to ensure accurate diagnosis of sarcoma in India. Our data shows alarming discrepancy of $60.8 \%$ in the histopathological diagnosis outside tertiary care centre. This discrepancy is certainly more than that stated in western literature (ranging 25-40\%) in various reports [6-11-12]. In a similar report from Pakistan [13], major discrepancy on second review (which had potential to change treatment) was $35.2 \%$ while minor discrepancy was $23.5 \%$ which is very similar to what we have reported (37.1\% and $23.7 \%$ respectively). We had no cases that were reported malignant outside but turned out to be benign unlike other reports where they exist in the tune of $5 \%$. Besides, we had 6 cases $(6.1 \%)$ that were referred to the other department as benign and found to have sarcoma on institutional review.

Though we have divided discrepancy into major and minor discrepancy according to decision change in individual patient, yet even minor discrepancy can lead to decision change in different setting. For example, in adjuvant setting large high-grade myxoid liposarcoma will have same treatment as pleomorphic undifferentiated sarcoma but in metastatic setting the treatment of myxoid liposarcoma includes promising newer drugs like trabectidin and eribulin which physician might not use in PUS. Thus, even minor discrepancy can't be taken trivially as it might altogether affect the treatment strategy. 
The frequency of major discrepancy was significantly higher in non-extremity sarcoma and this could be because pathologist and clinicians don't suspect sarcoma in visceral sites. To the best of our knowledge, this has not been previously reported in literature and might be useful indicator for guiding second review in resource constrained setting.

We believe in time to come this discrepancy can be lessened by reviewing histopathology in tertiary care centre as a part of treatment paradigm in soft tissue sarcoma. Given the population of India and numerically high burden of patients with sarcoma, Indian government must focus on opening dedicated sarcoma units in tertiary care centres. Even though sarcoma is a rare disease but the sheer volume of patients having this disease certainly deserve attention. Misdiagnosis of tumors like GIST also needs attention as GIST has good outcomes even in metastatic settings as compared to other soft tissue sarcomas. As of now, there is no standard guidelines in Indian setting however looking at guidelines issued by various expert groups we must incorporate those for our nation as well. The Guidance on Cancer Services issued by the National Institute for Health and Clinical Excellence (NICE) for soft tissue sarcoma explicitly state that all pathology should be either first reported or reviewed by a specialist soft tissue sarcoma pathologist [14].

We also believe that the unavailability of immunohistochemistry or the cost of immunohistochemistry is probably not the limiting factor as of patients who had immunohistochemistry report from outside $(n=27), 9$ patients still had major discrepancy, further reinforcing the fact that immunohistochemistry is not a good substitute for expert pathology. We didn't have data for the translocation tests for the study as it is not done in our centre and was done in case to case basis at the discretion of the physicians.

\section{Acknowledgements}

We thank the medical and administrative staff of All India Institute Of Medical Sciences, New Delhi for the support during the period of the study.

\section{Funding}

The study was not funded.

\section{References}

1. NCCN Clinical Practice Guidelines in Oncology [Internet]. [cited 2017 Dec 3]. Available from: https://www.nccn.org/ professionals/physician_gls/default.aspx

2. Husain N, Verma N. Curent Concepts in Pathology of Soft Tissue Sarcoma. Indian J Surg Oncol. 2011 Dec;2(4):302-6.

3. Ray-Coquard I, Thiesse P, Ranchère-Vince $D$, Chauvin $F$, Bobin J-Y, Sunyach M-P, et al. Conformity to clinical practice guidelines, multidisciplinary management and outcome of treatment for soft tissue sarcomas. Ann Oncol Off J Eur Soc Med Oncol. 2004 Feb;15(2):307-15.

4. Pisters PW, Leung DH, Woodruff J, Shi W, Brennan MF (1996). Analysis of prognostic factors in 1,041 patients with localized soft tissue sarcomas of the extremities. J Clin
Oncol, 14(5), 1679-89.

5. Gustafson P, Dreinhöfer KE, Rydholm A. Soft tissue sarcoma should be treated at a tumor center. A comparison of quality of surgery in 375 patients. Acta Orthop Scand. 1994 Feb;65(1):47-50.

6. Thway K, Fisher C (2009). Histopathological Diagnostic Discrepancies in Soft Tissue Tumours Referred to a Specialist Centre. Sarcoma, 2009, Article ID 741975, 7 pages.

7. Presant CA, Russell WO, Alexander RW, Fu YS. Soft-tissue and bone sarcoma histopathology peer review: the frequency of disagreement in diagnosis and the need for second pathology opinions. The Southeastern Cancer Study Group experience. J Clin Oncol. 1986 Nov 1;4(11):1658-61.

8. Arbiser ZK, Folpe AL, Weiss SW. Consultative (Expert) Second Opinions in Soft Tissue PathologyAnalysis of Problem-Prone Diagnostic Situations. Am J Clin Pathol. 2001 Oct 1;116(4):473-6.

9. Fisher C. Diagnosing sarcomas: the role of experience. Diagn Histopathol. 2011 Aug 1;17(8):333-9.

10. Dangoor A, Seddon B, Gerrand C, Grimer R, Whelan J, Judson I. UK guidelines for the management of soft tissue sarcomas. Clin Sarcoma Res. 2016 Nov 15;6:20.

11. Meis-Kindblom JM, Bjerkehage B, Böhling T, Domanski H, Halvorsen TB, Larsson O, et al. Morphologic review of 1000 soft tissue sarcomas from the Scandinavian Sarcoma Group (SSG) Register. The peer-review committee experience. Acta Orthop Scand Suppl. 1999 Jun;285:18-26.

12. Randall RL, Bruckner JD, Papenhausen MD, Thurman T, Conrad EU. Errors in diagnosis and margin determination of soft-tissue sarcomas initially treated at non-tertiary centers. Orthopedics. 2004 Feb;27(2):209-12.

13. Sharif MA, Hamdani SNR. Second opinion and discrepancy in the diagnosis of soft tissue lesions at surgical pathology. Indian J Pathol Microbiol. 2010 Jul 1;53(3):460.

14. Improving outcomes for people with sarcoma $\mid$ Guidance and guidelines | NICE [Internet]. [cited 2017 Dec 3]. Available from: ttps://www.nice.org.uk/guidance/csg9.

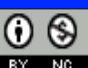

This work is licensed under a Creative Commons AttributionNon Commercial 4.0 International License. 